\title{
Cytokeratin 8 ectoplasmic domain binds urokinase-type plasminogen activator to breast tumor cells and modulates their adhesion, growth and invasiveness Nataša Obermajer ${ }^{1,2}$, Bojan Doljak ${ }^{2}$ and Janko Kos*1,2
}

Address: '1ožef Stefan Institute, Department of Biotechnology, Jamova 39, 1000 Ljubljana, Slovenia and ${ }^{2}$ University of Ljubljana, Faculty of Pharmacy, Aškerèeva 7, 1000 Ljubljana, Slovenia

Email: Nataša Obermajer - natasa.obermajer@ffa.uni-lj.si; Bojan Doljak - b.doljak@ffa.uni-lj.si; Janko Kos* - janko.kos@ffa.uni-lj.si

* Corresponding author

Published: 21 October 2009

Molecular Cancer 2009, 8:88 doi:10.1 186/1476-4598-8-88
Received: 14 July 2009

Accepted: 21 October 2009

This article is available from: http://www.molecular-cancer.com/content/8/I/88

(C) 2009 Obermajer et al; licensee BioMed Central Ltd.

This is an Open Access article distributed under the terms of the Creative Commons Attribution License (http://creativecommons.org/licenses/by/2.0), which permits unrestricted use, distribution, and reproduction in any medium, provided the original work is properly cited.

\begin{abstract}
Background: Generation of plasmin is a characteristic of tumor cells, promoting the degradation of extracellular matrix, tumor progression and metastasis. The process is accelerated if plasminogen and plasminogen activator are bound to their cell surface receptors.

Results: In this study we show that the monoclonal antibody that recognizes an epitope on the cytokeratin 8 (CK8) ectoplasmic domain (anti-CK MAb) inhibits plasminogen activation mediated by urokinase-type plasminogen activator (uPA) in MCF-7 and MCF-IOA neoT cells. The ectoplasmic domain of CK8 acts as a binding site for plasminogen, however, by using confocal microscopy, we demonstrated that it is also co-localized with uPA. CK8, therefore, function also as a receptor for uPA on the cell surface, and the presence of anti-CK MAb may prevent the binding of uPA to a designated CK8 motif. The consequent inhibition of plasmin generation resulted in changed cell morphology, enhanced cell adhesion to fibronectin, reduced invasion potential, and an enhanced G I/S transition. Moreover, surface plasmon resonance analysis showed that the synthetic dodecapeptide corresponding to the epitope sequence (VKIALEVEIATY), binds uPA in the nanomolar range.

Conclusion: These novel findings suggest a model in which CK8, together with uPA, plasminogen and fibronectin, constitutes a signaling platform capable of modulating cell adhesion/growthdependent signal transduction in breast tumor cells. Anti-CK MAb, which competes for the binding site for UPA, could be used as an agent to reduce the invasive potential of breast tumor cells.
\end{abstract}

\section{Background}

Proteases are essential for invasion by tumor cells. For this, they need to be activated, either on the tumor cell surface or in the pericellular space. In breast cancer, urokinase type plasminogen activator (uPA), a serine proteinase, has been associated with an aggressive malignant phenotype [1,2]. Cell surface uPA, bound to uPA receptor (UPAR), activates plasminogen to plasmin, a central player in breast cancer progression and metastasis. Plasmin activates growth factors and protease cascades that lead to the disruption of cell-cell and cell-extracellular matrix adhesion via pericellular proteolysis of glycopro- 
teins $[3,4]$. Plasmin can also affect the cell phenotype by activating or inactivating growth factors, and by modifying growth factor receptors and adhesion receptors [5-7].

Plasminogen is activated on the cell surface much faster than in solution, due to the presence of proteins that promote a plasminogen conformation more open to proteolytic attack [8]. In addition to uPAR, cytokeratin 8 (CK8) is an important plasminogen-binding protein in the membrane of breast cancer cells. CK8 is an intermediate filament protein and associates with CK18 to form an insoluble matrix within the cell. However, the C-terminal end of CK8 also penetrates the cellular membrane, as shown for hepatocellular and breast carcinoma cells $[9,10]$. CK8 is unique among cytokeratins in that it contains a carboxyl-terminal lysine that can interact with the lysine binding sites of plasminogen, although it is able to bind plasminogen even when the C-terminal lysine is mutated [11]. Plasminogen activation is promoted by tissue type plasminogen activator (tPA), which can bind to both components of the cytokeratin heterodimer, CK8/ CK18. Plasminogen and tPA cannot bind to CK8 at the same time, but the binding of tPA to CK18 enhances the binding of plasminogen to CK8 and its activation [11].

The CK8/CK18 complex modulates the signaling pathways intracellularly by binding kinases involved in signal transduction. It integrates signals generated by stimulated surface membrane receptors, such as insulin receptor and integrin beta 1 receptor, and modulates their signal transduction in appropriate reaction sequences. The absence of CK8/CK18 in hepatocytes results in a phenotype that attaches more rapidly to fibronectin and undergoes an enhanced G1/S transition compared with wild-type hepatocytes [12]. On the other hand, the cytoskeleton itself can undergo rearrangements as a result of outside-in signals triggered by fibronectin binding to cell surface receptors, the integrins.

The aim of this study was to investigate the involvement of CK8 in plasminogen activation, and consequently in cell adhesion, invasion and signaling of breast tumor cells. An anti-cytokeratin MAb directed against an epitope on the ectoplasmic tail of CK8 was used to determine whether it prevents uPA mediated activation of plasminogen, resulting in enhanced adhesion of breast tumor cells to fibronectin, reduced invasion, as well as changed morphology and enhanced G1/S transition.

\section{Results}

Plasminogen expression in breast tumor cells

Plasminogen was detected by flow cytometry in MCF-10 A neoT cells that were cultured in serum-free medium (Figure 1A). Staining with anti-plasminogen antibody showed the presence of plasminogen on the plasma mem- brane in non-permeabilized MCF-10 A neoT cells (Figure $1 \mathrm{~B})$. The staining pattern of plasminogen on the plasma membrane of non-permeabilized MCF cells was shown, by confocal microscopy, to be similar to that obtained with anti-CK MAb [13]. Observation of several confocal planes confirms that the staining follows the surface of the cell.

\section{uPA localization in breast tumor cells}

The treatment of MCF10A neoT cells with anti-CK MAb markedly affected breast tumor cell morphology. Whereas non-treated cells, grown on a fibronectin-coated surface, did not form typical epitheloid morphology but floated in clusters in the medium, anti-CK MAb treated breast tumor cells spread over the fibronectin-coated surface accompanied by rearrangement of the cytoskeleton, as evident by the reorganization of actin in bundles (Figures 2A and 2B).

uPA was co-localized with cytokeratins, detected with anti-CK MAb, in MCF-10A neoT cells cultured on fibronectin, which were not spread on the substratum but loosely attached in a cell cluster. On the other hand, $\mathrm{uPa}$ and cytokeratins were not co-localized in MCF-10A neoT cells that were firmly attached to the substratum and that exhibited epitheloid morphology (Figures 2C and 2D).

\section{Binding of uPA to a cytokeratin sequence recognized by anti-CK MAb}

The binding of uPA and plasminogen to the peptide VKIALEVEIATY, deduced from the C-terminal region of CK1, CK2, CK8, VK10, and recognized by anti-CK MAb [13], was assessed by surface plasmon resonance analysis. While the binding of plasminogen to the surface with immobilized peptide was not significantly different from that to the control flow cell surface lacking the peptide (data not shown), uPA bound specifically to the peptide in a concentration dependent manner, with a $K_{\mathrm{a}}$ of $9.8 \mathrm{nM}$ (Figure 3).

Additionally, the binding of UPA and anti-CK MAb was tested simultaneously in ELISA on immobilized peptide VKIALEVEIATY. The binding of anti-CK MAb was followed by the secondary anti-mouse antibody, conjugated to horse radish peroxidase or fluorescence dye Alexa Fluor 488. uPA and MAb competed for the binding to the peptide in a dose response manner (see additional file 1).

\section{Adhesion of breast tumor cells to fibronectin is increased by anti-CK MAb}

When MCF-10A neoT cells were seeded on fibronectin, they adhered more rapidly and to a greater extent in the presence of anti-CK MAb than in its absence (Figure 4). For instance, at 30 min post seeding, $67.7 \%$ of MCF-10A neoT cells were already attached in the presence of the 

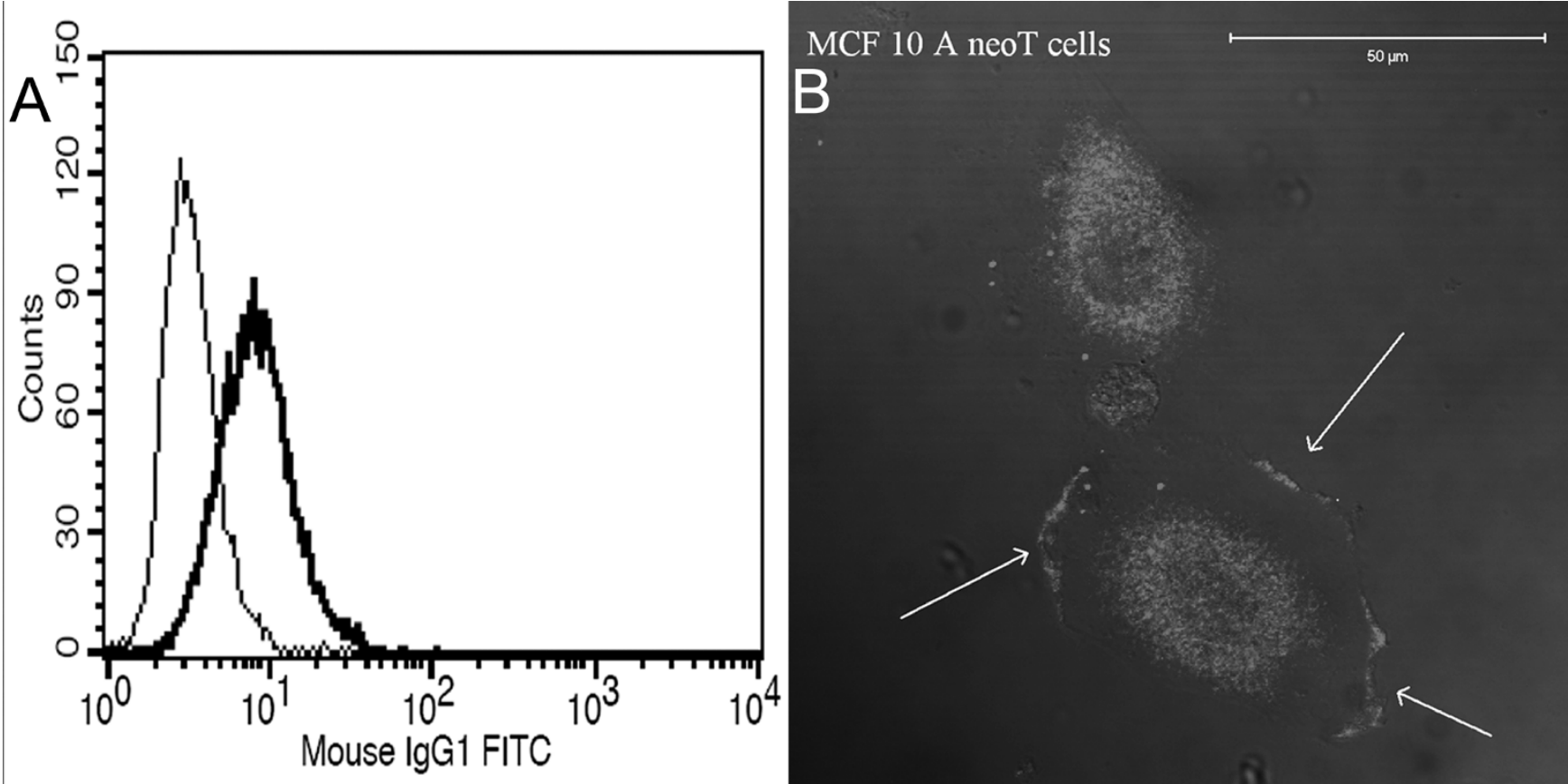

Figure I

Plasminogen expression and localization in MCF IOA neoT cells. (A) Flow cytometry histogram of MCF-IOA neoT cells stained with anti-plasminogen antibody. Surface expression of plasminogen was evaluated in MCFIOA neoT cells cultured on fibronectin in serum-free medium (bold line). The thin line represents the isotype control. (B) Plasminogen localization in MCF-IOA neoT cells. Non-permeabilized breast cancer cells cultured on fibronectin-coated glass coverslips were stained with anti-plasminogen antibody. Plasminogen is distributed on the surface of cells (pointing arrows).

anti-CK MAb as compared to $21.7 \%$ in its absence. The fact that the effect of anti-CK MAb was evident so quickly indicates that it acts by binding to the cell surface epitope and not by its intracellular activity. Even after $200 \mathrm{~min}$, only $48.8 \%$ of untreated cells were attached (Additional File 2), while anti-CK MAb treated MCF-10A neoT cells were all attached (Additional File 3). However, when uPA was added to the culture medium, the anti-CK MAb-stimulated adhesion of MCF-10A neoT cells was diminished (54.5\% after $200 \mathrm{~min}$ ), demonstrating that increased plasmin activity mediated by uPA reduces the adhesion of breast tumor cells to fibronectin. Anti-CK MAb did not alter cell adhesion to an uncoated surface, showing the substrate-dependent effect of the MAb (Figure 5).

\section{Invasion}

Anti-CK MAb was tested for its effect on the invasion of breast tumor cells through Matrigel. The addition of anti$\mathrm{CK}$ MAb to MCF-10A neoT cells strongly reduced the degree of invasion compared to control cells (Figure 6). This result can again be related to plasminogen activation on breast cancer cells. However, the viability and proliferation of breast tumor cells on Matrigel were not affected after $24 \mathrm{~h}$ by anti-CK MAb, as detected by MTS assay (data not shown).

\section{Effect of anti-CK MAb on the GI/S transition of breast tumor cells}

Breast tumor cells need to be seeded on a substratum in order to progress through the cell cycle in response to growth stimulation. On seeding MCF neoT cells on fibronectin in the presence of anti-CK MAb, more cells entered $S$ phase. The percentage of cells in $S$ phase, as detected by flow cytometry, was $4.2 \%$ for control cells and $10.0 \%$ for anti-CK MAb treated cells. In contrast, the G1/ $\mathrm{S}$ transition was not affected by anti-CK MAb when the cells were cultured on an uncoated surface (Figure 7A).

\section{Enhanced proliferation of breast tumor cells on fibronectin by anti-CK MAb}

The extent of breast tumor cell proliferation on fibronectin in the presence or absence of anti-CK MAb was assessed using the CFSE reagent. The CFSE proliferation assay does not depend on the metabolic activity of breast tumor cells and enables detection of cells that have undergone cell division. The addition of anti-CK MAb enhanced the proliferation of cells cultured on fibronectin from $77.3 \%$ to $94.2 \%$ (MCF10A neoT cells) and from $54.8 \%$ to $87.3 \%$ (MCF-7 cells). No enhancement was observed on surfaces not covered with fibronectin (Figure 7B). 

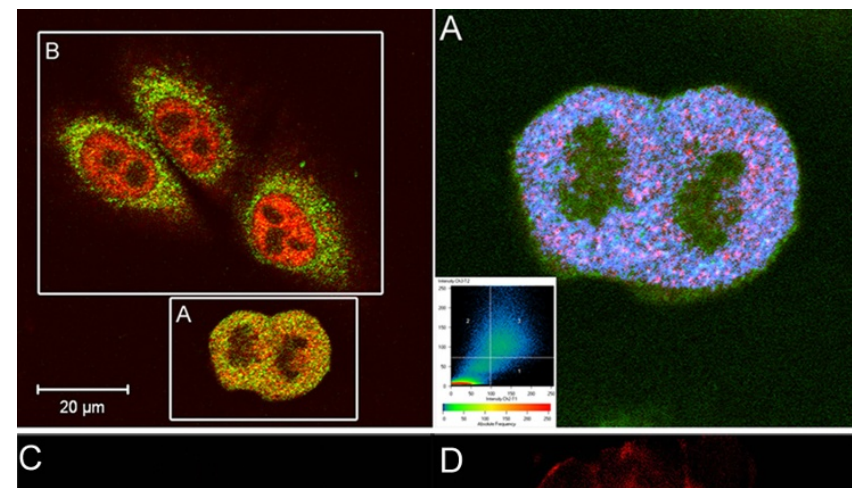

D

\section{(1)}

\section{Figure 2}

Localization of uPA, cytokeratin and actin in MCFIOA neoT cells. Colocalization of anti-uPA antibody and anti-CK MAb (A). MCF-IOA neoT cells were stained with anti-uPA MAb (green fluorescence, Alexa-488) and antiCK MAb (red fluorescence, Alexa-555) and imaged sequentially in a line-interlace mode to eliminate cross talk between the channels. The threshold level for this display was set arbitrarily. Pixels above the threshold in both channels (blue color, right panel) and the contour plot are shown for MCFIOA neoT cell cluster (inset C) demonstrating strong co-localization in contrast to adherent MCF-IOA neoT cells (B). Anti-CK Mab triggered actin reorganization in MCF-IOA neoT cells. MCF-IOA neoT cells, treated (C) and nontreated (D) with anti-CK MAb, were cultured on fibronectin for $2 \mathrm{~h}$ and stained with phalloidin. Actin bundles are seen in anti-CK8 MAb treated cells but not in non-treated cells.

\section{Discussion}

We have demonstrated that uPA is able to bind a particular motif on the CK8/CK18 complex expressed on the plasma membrane of breast tumor cells, that is recognized also by anti-CK MAb. The antibody hinders uPA binding to the cell surface of MCF-7 and MCF-10A neoT breast cells [13], resulting in lower plasmin generation, adhesion, invasiveness and G1/S transition of breast tumor cells.

Although plasminogen does not bind to the motif that resembles the epitope of anti-CK MAb (data not shown), anti-CK MAb significantly reduces plasminogen activation in MCF-7 and MCF-10A neoT cells [13]. Plasminogen is shown to be expressed endogenously in these cells and localized intracellularly as well as on the plasma mem- brane. The activation of plasminogen to plasmin enables degradation of the proteins of the extracellular matrix, a process that enhances the invasive potential of tumor cells. Plasminogen activation is enhanced if plasminogen activator (uPA/tPA) and the substrate (plasminogen) are in close juxtaposition on the cell surface. The most prominent uPA binding receptor is UPAR (CD87). However, plasminogen activation independent of uPAR suggests that other uPA binding proteins exist on the plasma membrane. On neutrophils, for example, $\alpha_{M} \beta_{2}$ integrin receptor binds UPA irrespectively of the presence of UPAR [14]. In this case, UPAR may serve as an enhancer of $\alpha_{M} \beta_{2}$ binding, and uPAR, uPA, $\alpha_{M} \beta_{2}$ and plasminogen may form a multicontact complex on the cell surface. MCF-7 cells, for example, express very low levels of cell surface uPAR compared to the larger amount of cell surface bound plasminogen [15]. Our results show that CK8, the major plasminogen receptor on breast tumor cells [16], may also serve as a uPA binding protein and enhance uPA mediated plasminogen activation. As shown by surface plasmon resonance analysis, the interaction between $\mathrm{UPA}$ and CK8 is significant, since uPA binds the peptide VKIALEVEIATY, deduced from the C-terminal region of $\mathrm{CK} 8$, in the nanomolar range ( $K_{\mathrm{a}}$ of $9.8 \mathrm{nM}$ ). This peptide, which also binds strongly to the anti-CK MAb, contains a consensus sequence, corresponding to the most conserved region between D381 and E407 (CK8) in CK1, CK2, CK8, CK10 and CK18. Anti-CK MAb was shown to recognize these CKs, therefore, we conclude that the epitope is identical, independently of the exact amino acid sequence [13].

Expression of CK8 on the cell surface correlates with increased invasiveness in vitro and in vivo $[17,18]$ and may predispose cell polarization and adhesion [19]. We have shown that anti-CK MAb, by diminishing the uPA mediated plasminogen activation in serum-free environment [13], affects the de-adhesion-invasion processes of breast tumor cells. The adhesion of these cells treated with antiCK MAb was greatly enhanced, due to the reduced pericellular proteolysis of fibronectin, increasingly degraded in the tumor environment by plasmin. This is consistent with the findings of Tan et al. who showed that plasminogen expression, restricted to cells with high invasion ability, is involved in cell dissociation, the first important step in the invasion process [20]. The adhesion of MCF cells to fibronectin is mediated via $\beta_{1}$ integrin receptors, which can be modified by plasmin in a way that affects the cell phenotype $[7,21]$. Although we cannot exclude a direct effect of plasmin on integrin chains, we suggest that the anti-adhesive effect of plasmin results from the proteolytic cleavage of fibronectin, similarly to the way that plasmin, when generated by the uPA-dependent cell surface-associated pathway, abrogates the $\mathrm{HaCaT}$ cell binding functions via cleavage of vitronectin and destruction of its cell binding function [22]. In contrast, anti-CK MAb did not affect cell adhesion to the surface not coated with fibronectin. 


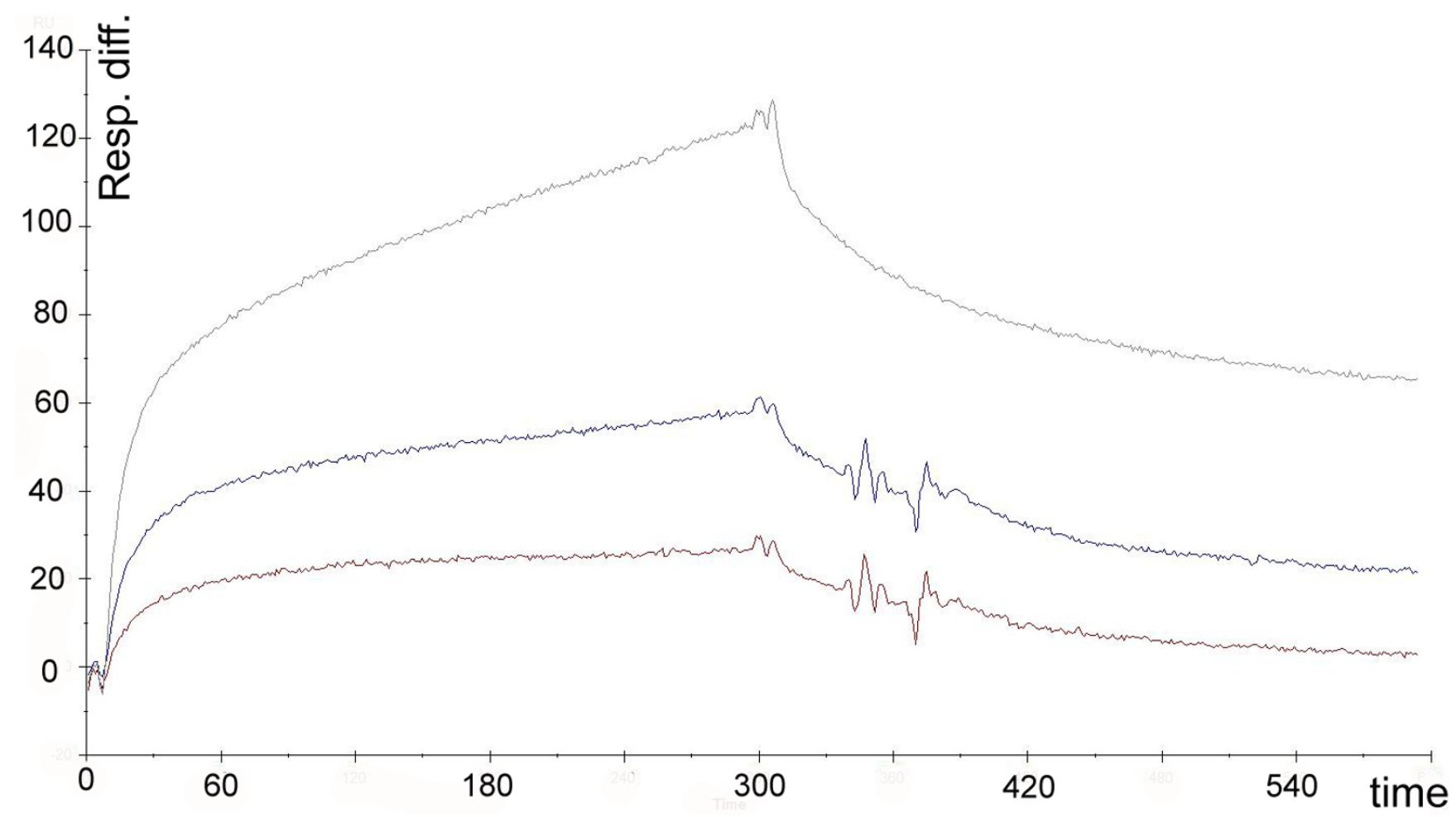

Figure 3

uPA binding to dodecapeptide VKIALEVEIATY. Association and dissociation curves of uPA binding to VKIALEVEIATY peptide, obtained by surface plasmon resonance (from the bottom up, uPA at $10 \mathrm{nM}, 20 \mathrm{nM}$ and $50 \mathrm{nM}$ ). A $K_{\mathrm{a}}$ of $9.8 \mathrm{nM}$ was calculated, using the I:I binding with drifting baseline model.

Our results further show that, in breast tumor cells cultured on fibronectin in serum free medium, anti-CK MAb reduces cell cluster formation and promotes cell polarization associated with actin reorganization. This is consistent with the observations that, in the presence of uPA or plasmin, MCF-7 breast tumor cells begin to retract and form solid tumor cell spheroids [23]. The spontaneous formation of the spheroids starts after supplementing the culture medium with an outdated source of human serum containing uPA and plasminogen [23].

Anti-CK MAb treated breast tumor cells cultured on fibronectin, but not on uncoated surface, also exhibited enhanced proliferation. The latter can be attributed to an increased mitogenic signal delivered by fibronectin, where the activating signal is mediated by $\beta_{1}$ integrin receptor. The signaling reactions activated by $\beta_{1}$ integrins include focal adhesion kinase (FAK)-Src and MAPK pathways, and generate sustained activation of the PI3K-Akt pathway, a property that could be important for anchorage-dependent survival of cells [24]. MCF-7 cell-fibronectin interaction induces the re-entry of MCF-7 cells into $S$ phase, and prevents them from undergoing serum deprivation induced apoptosis [25]. Anti-CK MAb increases adhesion of breast cancer cells to fibronectin, thereby promoting their G1/S transition. These novel findings suggest a model by which CK8, along with plasminogen-see earlier comment and fibronectin, constitutes a signaling platform capable of modulating cell adhesion/growth-dependent signal transduction. A similar effect was observed in CK8null hepatocytes, which attach more rapidly but spread more slowly on a fibronectin substratum. Loss of CK8/ CK18 also leads to decreased size, reduced rate of protein synthesis, and an enhanced G1/S transition in hepatocytes [12]. The analogy between hepatocytes and the MCF-7/MCF-10A neoT used in our experiments is not surprising, considering that CK8-mediated plasmin activation has been reported on hepatocytes and hepatocellular carcinoma cells, as well as on certain breast tumor cells [19].

\section{Conclusion}

Anti-CK MAb, that recognizes the epitope on the ectoplasmic tail of CK8 present on the plasma membrane of the breast tumor cells, prevents the binding of uPA to cytokeratin and, consequently, the activation of plasminogen to plasmin. Anti-CK MAb could thus be used as an agent to reduce the invasive potential of breast tumor cells, 


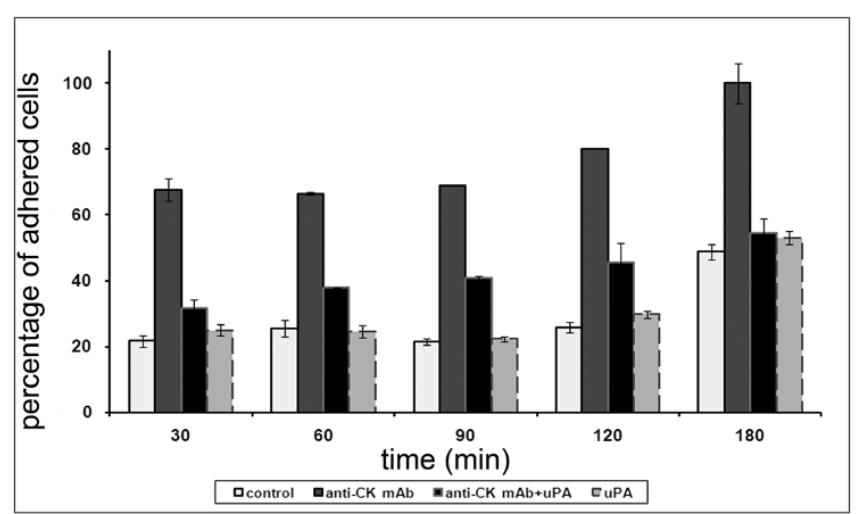

Figure 4

Adhesion of MCF-I OA neoT cells to fibronectin in the presence of anti-CK MAb and uPA. MCFIOA neoT cells were cultured on a fibronectin coated surface in the absence or presence of anti-CK MAb and/or uPA. The percentage of adherent cells was assessed with MTS assay. The time course of adhesion to the fibronectin surface is shown (See also Video SI and S2). Samples were analyzed in quadruplicate and each point is the mean of at least three independent experiments.

whereas the synthetic peptide, resembling the epitope, may regulate uPA activity.

\section{Methods \\ Cell culture}

MCF-10A neoT cells were provided by Prof. Bonnie F. Sloane (Wayne State University, Detroit, MI). The original human breast epithelial cell line (MCF-10) was transformed with neomycin resistance gene and c-Ha-ras oncogene, to give a highly invasive cell phenotype. MCF-7 cells were obtained from ATCC (НTB 22). Cells were cultured in monolayers to $80 \%$ confluence in DMEM/F12 medium supplemented with $12.5 \mathrm{mM}$ HEPES, $2 \mathrm{mM}$ glutamine, $5 \%$ fetal bovine serum (FCS), insulin, hydrocortisone, epidermal growth factor and antibiotics at $37^{\circ} \mathrm{C}$ in a humidified atmosphere containing $5 \% \mathrm{CO}_{2}$. The cells were maintained in serum-free medium for $24 \mathrm{~h}$ before being used in assays. Cells were washed with PBS, pH 7.4 and detached from culture flasks with $0.05 \%$ trypsin and $0.02 \%$ EDTA in PBS, pH 7.4. The viability of cells in the experiments was at least $90 \%$, as determined by staining with nigrosin.

\section{Antibody preparation}

Anti-CK MAb, a mouse monoclonal antibody, was raised against soluble membrane proteins of MCF-7 human invasive ductal breast carcinoma. Using immunocytochemical analysis, its staining was detected predominantly in primary breast carcinomas and in metastatic lymph nodes $[13,26]$. The antibody recognizes a specific cytokeratin profile (cytokeratins 1, 2, 8, 10, 18) in MCF-7 and MCF-10A neoT breast cancer cell lines [13], as determined by $2 \mathrm{D}$ electrophoresis, immunoblotting and mass spectroscopy. Hybridoma cells for antibody isolation were cultured in DMEM medium supplemented with 2 $\mathrm{mM}$ glutamine, $13 \%$ FCS and antibiotics, at $37^{\circ} \mathrm{C}$ in a humidified atmosphere containing $5 \% \mathrm{CO}_{2}$. The antibody was isolated by affinity chromatography on Protein A Sepharose using standard procedures. On MCF-7 and MCF-10A neoT it was demonstrated that the antibody inhibited the generation of plasmin in a dose dependent manner [13].

\section{Immunofluorescence}

For flow cytometry, MCF-10A neoT cells were grown for 24 hours in serum free medium and afterwards cultured on 12-well plates pre-coated with fibronectin (Corning Costar). Cells were harvested and fixed in $4 \%$ paraformaldehyde for $30 \mathrm{~min}$, and permeabilized for 10 minutes with Triton-X 100. Non-specific staining was blocked for 30 minutes with $3 \%$ BSA in phosphate buffered saline (PBS), pH 7.4. Cells were incubated with $5 \mu \mathrm{g} / \mathrm{mL}$ goat anti-plasminogen polyclonal antibody in blocking buffer for 1 hour on ice. Afterwards, the cells were washed with PBS and incubated with Alexa-488 labeled anti-goat secondary antibody in blocking buffer for 1 hour on ice. Alexa-488-IgG1 was used for isotype control. Cells were washed and analyzed with FACS Calibur (Becton Dickinson, NJ).

For confocal microscopy, glass coverslips were pre-treated with $20 \%$ sulfuric acid, followed by $10 \mathrm{M} \mathrm{NaOH}$, and washed with distilled water and ethanol before use. They were coated with $1 \mu \mathrm{g} / \mathrm{mL}$ fibronectin in carbonate buffer, $\mathrm{pH} 9.6$ overnight at $4{ }^{\circ} \mathrm{C}$. For immunofluorescence detection, MCF-10A neoT cells were cultured on either fibronectin-coated or uncoated coverslips for 24 hours. Cells were fixed with $2 \%$ paraformaldehyde at room temperature for 40 minutes, then permeabilized with $0.05 \%$ Triton-X 100. To preserve membrane associated components and foreclose cytoplasmic staining, some samples were not permeabilized with Triton-X 100. Non-specific staining was blocked with 3\% BSA in phosphate buffer saline (PBS), pH 7.4, for 1 hour. After 1.5 hour of incubation with anti-CK MAb and either rabbit anti-uPA polyclonal antibody or goat anti-plasmin(ogen) polyclonal antibody (Santa Cruz Biotechnology, CA), cells were washed with PBS. Secondary Alexa-488 labeled anti-rabbit or Alexa-488 labeled anti-goat and Alexa-555 labeled anti-mouse antibody were incubated for 1 hour in blocking buffer and afterwards washed with PBS. Actin was labeled with phalloidin-tetramethylrhodamine B isothiocyanate conjugate (Fluka) $(500 \mathrm{ng} / \mathrm{mL})$ for $30 \mathrm{~min}$ at room temperature in anti-CK MAb treated or un-treated 


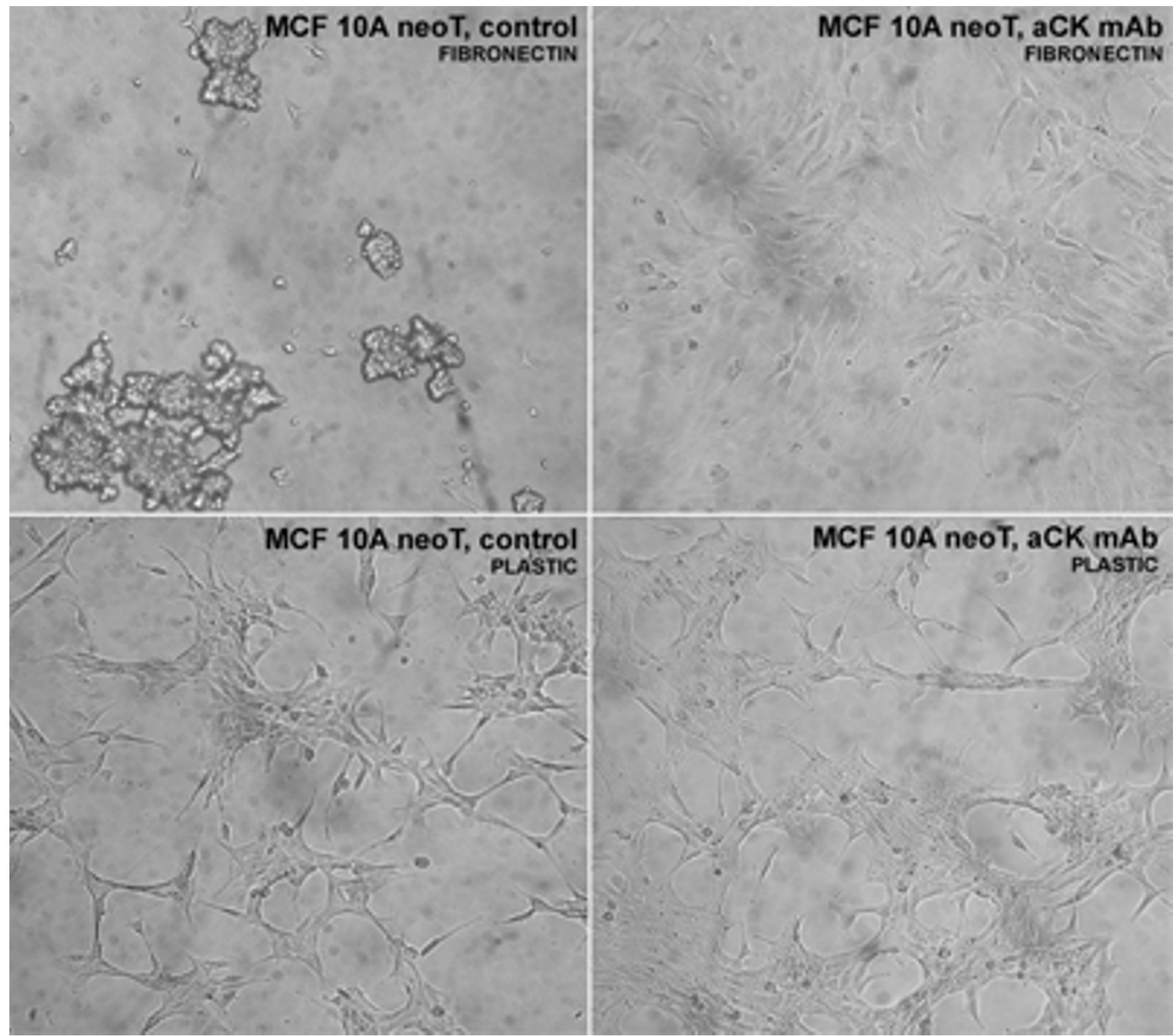

\section{Figure 5}

Anti-CK MAb changes the morphology of MCF-I OA neoT cells cultured on fibronectin. Photomicrographs of MCF-IOA neoT cells cultured for $24 \mathrm{~h}$ on uncoated (below) or fibronectin-coated surfaces (above) are shown for control and anti-CK8 MAb treated cells. Anti-CK MAb enables the adhesion to fibronectin of MCF-IOA neoT cells, but not control cells, which form cell clusters. In contrast, both control and anti-CK MAb treated cells adhere to uncoated surface.

cells, then washed with PBS. Prolong Antifade kit (Molecular Probes, CA) was used for mounting coverslips on glass slides. Fluorescence microscopy was performed using a Carl Zeiss LSM 510 confocal microscope. Alexa 488 and FITC were excited with an argon (488 nm) laser and Alexa 546 with a $\mathrm{He} / \mathrm{Ne}(543 \mathrm{~nm})$ laser. Emission was filtered using narrow band 505-530 nm or LP $560 \mathrm{~nm}$ filters, respectively. Images were analyzed using Carl Zeiss LSM image software 3.0.

\section{Surface plasmon resonance}

The binding of uPA to the peptide VKIALEVEIATY (Doljak et al., 2008) was monitored using a Biacore $X$ system (Biacore, Sweden). The assay was performed on a CM5 sensor chip (BR-1003-98 BIAcore). VKIALEVEIATY was immobilized at a flow rate of $1 \mu \mathrm{L} / \mathrm{min}$ for 10 minutes (300 RU). The flow cell was then washed with $5 \mu \mathrm{L}$ of 10 $\mathrm{mM}$ glycine buffer ( $\mathrm{pH} 2.2$ ) at a flow rate of $30 \mu \mathrm{L} / \mathrm{min}$. The second flow cell was used as the reference cell. Identical wash cycles were used to regenerate the dodecapeptide 


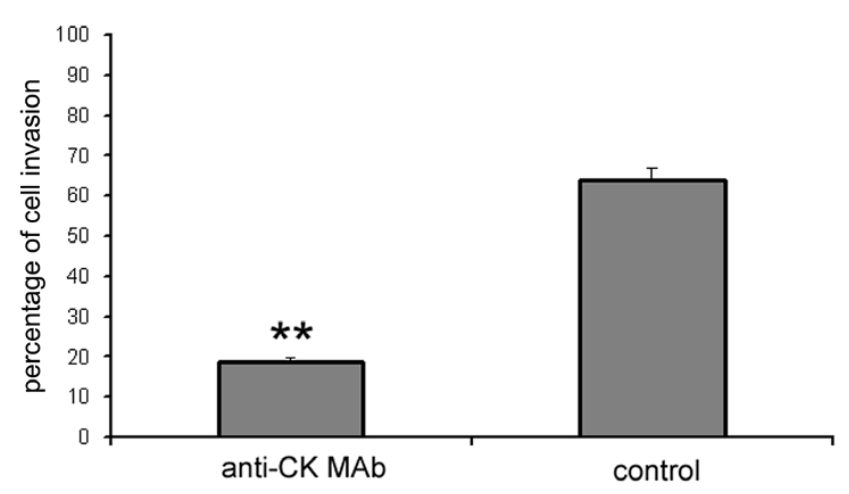

Figure 6

Anti-CK MAb reduces the invasiveness of MCF-IOA neoT cells. The degree of invasion of Matrigel by MCF-IOA neoT cells is shown in the presence and absence of anti-CK MAb. It is presented as the percentage of invasion of MCFIOA neoT cells to the lower compartment of a Transwell chamber. Bars represent mean percentages of migrated cells \pm s.d. $P$ values $<0.001$ are marked with $* *$.
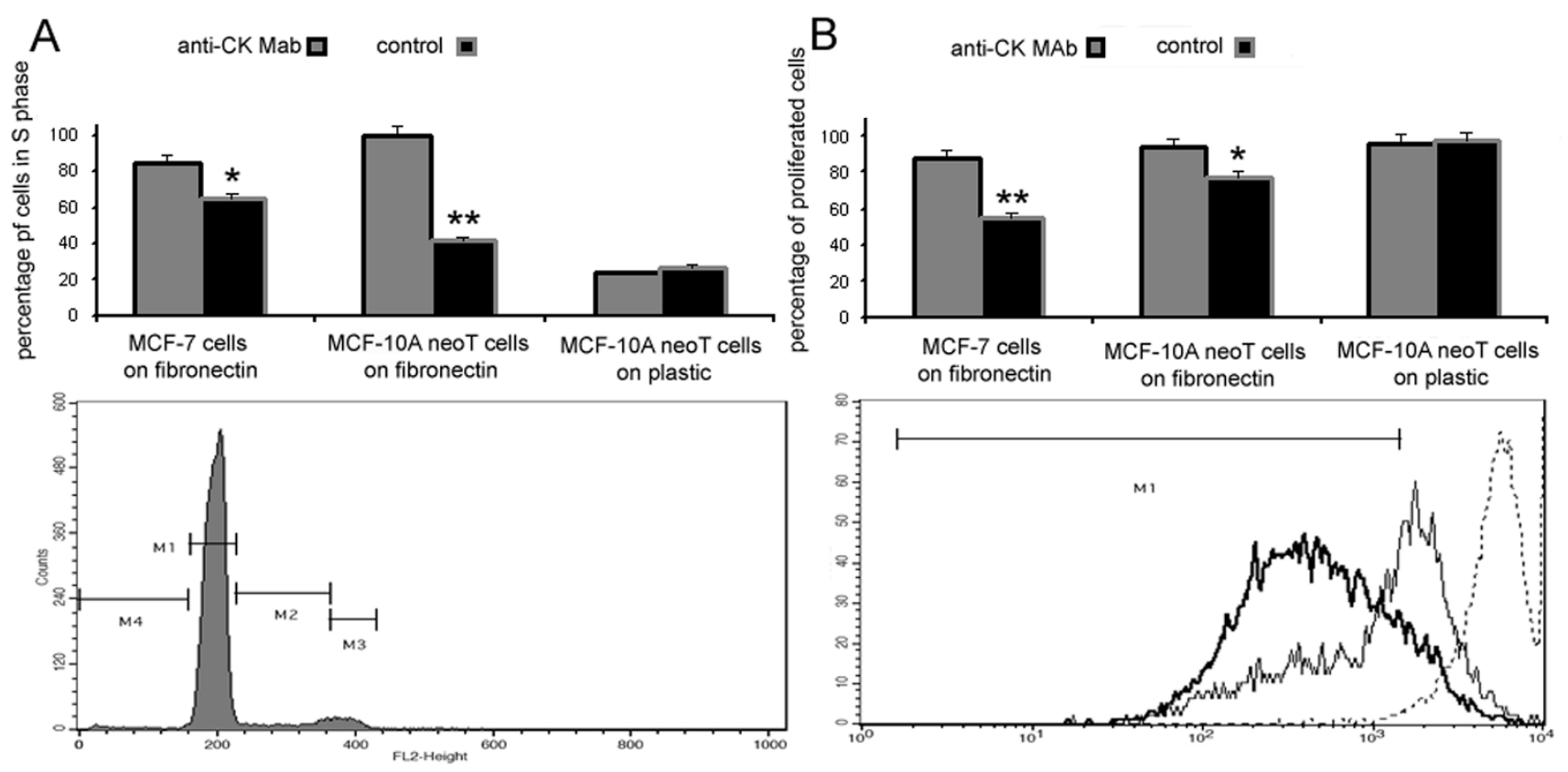

\section{Figure 7}

The effect of anti-CK MAb on cell cycle (A) and proliferation (B) of breast tumor cells cultured on fibronectin. MCF-IOA neoT and MCF-7 cells were cultured in serum-free medium in the absence or presence of anti-CK MAb. After $24 \mathrm{~h}$ they were harvested and analyzed for cell cycle status by flow cytometry. The percentage of anti-CK MAb treated and untreated cells in $S$ phase is given ( $A$, top). The representative histogram used to determine the percentage of distinctive cell populations is shown at the bottom. For the proliferation assay, MCF-7 cells were labeled with CFSE reagent and cultured for $48 \mathrm{~h}$ in the presence or absence of anti-CK MAb. Proliferation threshold was set according to CFSE labeled cells analyzed immediately after labeling (B, bottom). The percentage of proliferating cells of the PIneg population is given (B, top). Results represent at least three independent assays. $P$ values of $<0.05$ are marked with $*$, and $P$ values $<0.00$ I with $* *$. 
partments were washed with PBS and the cells detached with $0.05 \%$ trypsin and $0.02 \%$ EDTA in PBS, pH 7.4. Cells from the lower compartments were transferred separately to Eppendorf tubes and centrifuged at $1500 \mathrm{rpm}$ for 5 minutes. They were resuspended in culture medium and transferred to 96-well plates. The Cell Proliferation Assay CellTiter $96^{\circledR}$ One Solution from the (3-(4,5-dimethylthiazol-2-yl)-5-(3-carboxymethoxyphenyl)-2-(4-sulfophenyl)-2H-tetrazolium) MTS (colorimetric assay, Promega, WI) was added and the plate incubated for an additional 2 hours. The absorbance, A, of the formazan product was measured at $590 \mathrm{~nm}$. Invasion was recorded as the percentage of the cells that penetrated the Matrigel-coated filters:

$$
\text { Cell invasion }(\%)=\left(\mathrm{A}_{\text {invasive }} / \mathrm{A}_{\text {total }}\right) \times 100
$$

\section{Cell adhesion assay}

A 96-well culture plate was pre-coated with $50 \mu \mathrm{L}$ of fibronectin $(1 \mu \mathrm{g} / \mathrm{mL})$ in carbonate buffer, $\mathrm{pH}$ 9.6, overnight at $4^{\circ}$. Wells were then washed once with PBS and incubated with $1 \%$ BSA in PBS for $30 \mathrm{~min}$ at room temperature. MCF-10A neoT cells were harvested, washed with PBS and resuspended in the serum-free medium. Anti-CK MAb was added to the medium at a final concentration $1 \mu \mathrm{M}$. $50 \mu \mathrm{L}$ of MCF-10A neoT cell suspension was added to each well of a 96-well culture plate (TPP, Switzerland) pre-coated with fibronectin. Cells were allowed to attach for $15 \mathrm{~min}, 45 \mathrm{~min}, 1.5 \mathrm{~h}, 3 \mathrm{~h}$ and $5 \mathrm{~h}$, after which wells were washed twice with PBS, and $100 \mu \mathrm{L}$ of complete growth medium was added. The assay was performed in quadruplicate. Control wells were washed with $50 \mu \mathrm{L}$ of medium. Finally, CellTiter $96^{\circledR}$ One Solution was added, formazan absorbance measured, and cell adherence was calculated from the equation:

$$
\text { cell adherence }(\%)=\left(\mathrm{A}_{\mathrm{tx}} / \mathrm{A}_{\text {control }}\right) \times 100 \text {, }
$$

where the absorbance of formazan were determined for cells washed at different time points $\left(A_{t x}\right)$ and for cells not washed $\left(\mathrm{A}_{\text {control }}\right)$.

Differential interference contrast images were taken with an Olympus IX 81 motorized inverted microscope and Cell ${ }^{\circledR}$ software.

\section{Cell cycle analysis}

A 12-well culture plate was pre-coated with $250 \mu \mathrm{L}$ of fibronectin $(1 \mu \mathrm{g} / \mathrm{mL})$ in carbonate buffer, $\mathrm{pH} 9.6$ overnight at $4{ }^{\circ} \mathrm{C}$. Wells were washed once with PBS and incubated with $1 \%$ BSA in PBS for $30 \mathrm{~min}$ at room temperature. MCF-10A neoT cells were harvested, washed with PBS and resuspended in the serum-free medium in the presence of anti-CK MAb $(1 \mu \mathrm{M})$, while the antibody was not added to control wells. MCF-10A neoT cells $(1 \times$
$10^{5}$ cells, $500 \mu \mathrm{L}$ ) were added to a 12-well culture plate (TPP) pre-coated with fibronectin. Control wells were not pre-coated with fibronectin. Cells were cultured for either $48 \mathrm{~h}$ or $72 \mathrm{~h}$, washed twice with PBS and detached with $0.05 \%$ trypsin and $0.02 \%$ EDTA in PBS, pH 7.4. Cells were centrifuged at $1500 \mathrm{rpm}$ for $5 \mathrm{~min}$, resuspended in icecold ethanol and incubated at $-20^{\circ} \mathrm{C}$ for $45 \mathrm{~min}$. Cells were then centrifuged again and propidium iodide and RNAse added to final concentrations of $40 \mu \mathrm{g} / \mathrm{ml}$ and $0.1 \%$. Cells were incubated for $30 \mathrm{~min}$ at $37^{\circ} \mathrm{C}$, washed with PBS and analyzed for DNA content. Flow cytometry was performed on a FACS Calibur (Becton Dickinson, NJ).

\section{Cell proliferation assay}

A 12-well culture plate was pre-coated overnight with 250 $\mu \mathrm{L}$ of fibronectin in carbonate buffer, $\mathrm{pH} 9.6(1 \mu \mathrm{g} / \mathrm{mL})$, at $4{ }^{\circ} \mathrm{C}$. Wells were washed once with PBS and incubated with $1 \%$ BSA in PBS for $30 \mathrm{~min}$ at room temperature. MCF-10A neoT cells were harvested, washed with PBS, labeled with CFSE (5-carboxyflourescein diacetate succinimidyl ester) cell tracing reagent (Molecular Probes, CA) according to the manufacturer's protocol, and re-suspended in the serum-free medium, with or without antiCK MAb $(1 \mu \mathrm{M})$. MCF-10A neoT cells $\left(1 \times 10^{5}\right.$ cells, 500 $\mu \mathrm{L}$ ) were added to a 12-well culture plate (TPP) pre-coated with fibronectin. Control wells were not pre-coated with fibronectin. Cells were cultured for either $48 \mathrm{~h}$ or $72 \mathrm{~h}$. Wells were then washed twice with PBS and cells detached with $0.05 \%$ trypsin and $0.02 \%$ EDTA in PBS, pH 7.4. The threshold for proliferating cells was set according to MCF10A neoT cells measured for CFSE fluorescence intensity at the beginning of the assay. Flow cytometry was performed on a FACS Calibur (Becton Dickinson).

\section{Statistical analysis}

SPSS PC software package (Release 13.0) was used for statistical analysis. The difference between the groups was evaluated using the non-parametric Mann-Whitney test. P values of $<0.05$ were considered to denote statistical significance.

\section{Competing interests}

The authors declare that they have no competing interests.

\section{Authors' contributions}

NO conceived the study and participated in its design, performed the experiments and drafted the manuscript. BD performed the experiments. JK participated in the coordination, supervised the study and helped to draft the manuscript. All authors read and approved the final manuscript. 


\section{Additional material}

\section{Additional file 1}

$S 1$ - Competition of anti-CK MAb and UPA for binding to immobilized peptide VKIALEVEIATY. Graph showing the competition of anti-CK $M A b$ and $u P A$ for binding to immobilized peptide VKIALEVEIATY. The concentration of anti-CK MAb was $6.7 \mathrm{nM}$. Secondary goat-antimouse antibody conjugated to HRP was used for the detection of bound anti-CK $M A b$.

Click here for file

[http://www.biomedcentral.com/content/supplementary/14764598-8-88-S1.TIFF]

\section{Additional file 2}

S2 - Adhesion of MCF-10A neoT cells to fibronectin in the absence of anti-CK MAb. Video showing adhesion of MCF-10A neoT cells to fibronectin in the absence of anti-CK MAb.

Click here for file

[http://www.biomedcentral.com/content/supplementary/14764598-8-88-S2.MOV]

\section{Additional file 3}

S3 - Adhesion of MCF-10A neoT cells to fibronectin in the presence of anti-CK MAb. Video showing the adhesion of MCF-10A neoT cells to fibronectin in the presence of anti-CK MAb.

Click here for file

[http://www.biomedcentral.com/content/supplementary/14764598-8-88-S3.MOV]

\section{Acknowledgements}

The authors thank Professor Roger Pain for critical reading of the manuscript. Surface plasmon resonance experiments were performed in the Infrastructural Center for Surface Plasmon Resonance at the Department of Biology, University of Ljubljana. We thank Ms. Astrid Strgaršek for excellent technical assistance. This work was supported by a grant from the Research Agency of the Republic of Slovenia (grant P4-0127 to JK) and partially by the Seventh EU Framework IP project NanoPhoto (JK).

\section{References}

I. Bianchi E, Cohen R, Thor A, Todd Rr, Mizukami I, Lawrence D, Ljung $B M$, Shuman MA, Smith HS: The urokinase receptor is expressed in invasive breast cancer but not in normal breast tissue. Cancer Res 1994, 54:86I-866.

2. Duffy M, Reilly D, O'Sullivan C, O'Higgins N, Fennelly J, Andreasen P: Urokinase-plasminogen activator, a new and independent prognostic marker in breast cancer. Cancer Res 1990, 50:6827-6829.

3. Reinartz J, Batrla R, Boukamp P, Fusenig N, Kramer M: Binding and activation of plasminogen at the surface of human keratinocytes. Exp Cell Res 1993, 208:197-208.

4. Geer $D$, Andreadis $S$ : A novel role of fibrin in epidermal healing. plasminogen-mediated migration and selective detachment of differentiated keratinocytes. J Invest Dermatol 2003, 121:1210-1216.

5. Gonias S, Young WJ, Fox J: Cleavage of recombinant murine interferon-gamma by plasmin and miniplasmin. J Interferon Res 1989, 9:517-529.

6. Lyons A, Ashman L: The effect of recombinant cytokines on the proliferative potential and phenotype of cells of the human myelomonocytic leukaemia line, RC-2A. Leuk Res 1988, 1 2:659-666.

7. Pasche B, Ouimet $\mathrm{H}$, Francis S, Loscalzo J: Structural changes in platelet glycoprotein IIb/IIla by plasmin: determinants and functional consequences. Blood I994, 83:404-4I4.
8. Ellis V, Behrendt N, Danø K: Plasminogen activation by receptor-bound urokinase. A kinetic study with both cell-associated and isolated receptor. J Biol Chem I991, 266: I 2752-I 2758.

9. Hembrough T, Vasudevan J, Allietta M, Glass WF II, Gonias S: A cytokeratin 8-like protein with plasminogen-binding activity is present on the external surfaces of hepatocytes, HepG2 cells and breast carcinoma cell lines. J Cell Sci 1995, 108: 107I-1082.

10. Ditzel H, Garrigues U, Andersen C, Larsen M, Garrigues H, Svejgaard A, Hellström I, Hellström KE, Jensenius JC: Modified of cytokeratins expressed on the surface of carcinoma cells undergo endocytosis upon binding human monoclonal antibody and its recombinant Fab fragment. Proc Natl Acad Sci USA 1997, 94:8II0-8II5.

II. Kralovich K, Li L, Hembrough T, Webb D, Karns L, Gonias S: Characterization of the binding sites for plasminogen and tissuetype plasminogen activator in cytokeratin 8 and cytokeratin 18. J Protein Chem 1998, 17:845-854.

12. Galarneau L, Loranger A, Gilbert S, Marceau N: Keratins modulate hepatic cell adhesion, size and GI/S transition. Exp Cell Res 2007, 31 3:179-194.

13. Doljak B, Obermajer N, Jamnik P, Kos J: Monoclonal antibody to cytokeratin VKIALEVEIATY sequence motif reduces plasminogen activation in breast tumour cells. Cancer Lett 2008, 267:75-84.

14. Pluskota E, Soloviev D, Bdeir K, Cines D, Plow E: Integrin alpha M beta2 orchestrates and accelerates plasminogen activation and fibrinolysis by neutrophils. J Biol Chem 2004, 279: |8063-18072.

15. Nguyen D, Hussaini I, Gonias S: Binding of urokinase-type plasminogen activator to its receptor in MCF-7 cells activates extracellular signal-regulated kinase $I$ and 2 which is required for increased cellular motility. J Biol Chem 1998, 273:8502-8507.

16. Hembrough T, Li L, Gonias S: Cell-surface cytokeratin 8 is the major plasminogen receptor on breast cancer cells and is required for the accelerated activation of cell-associated plasminogen by tissue-type plasminogen activator. J Biol Chem 1996, 27 1:25684-2569|.

17. Azumi N, Battifora $\mathrm{H}$ : The distribution of vimentin and keratin in epithelial and nonepithelial neoplasms. A comprehensive immunohistochemical study on formalin- and alcohol-fixed tumors. Am J Clin Pathol 1987, 88:286-296.

18. Miettinen M, Franssila K: Immunohistochemical spectrum of malignant melanoma. The common presence of keratins. Lab Invest 1989, 61:623-628.

19. Gonias S, Hembrough T, Sankovic M: Cytokeratin 8 functions as a major plasminogen receptor in select epithelial and carcinoma cells. Front Biosci 200I, 6:DI 403-I4II.

20. Tan X, Egami $H$, Nozawa F, Abe M, Baba $\mathrm{H}$ : Analysis of the invasion-metastasis mechanism in pancreatic cancer: involvement of plasmin(ogen) cascade proteins in the invasion of pancreatic cancer cells. Int J Oncol 2006, 28:369-374.

21. Lamarre J, Vasudevan J, Gonias S: Plasmin cleaves betaglycan and releases a $60 \mathrm{kDa}$ transforming growth factor-beta complex from the cell surface. Biochem J 1994, 302:199-205.

22. Reinartz J, Schäfer B, Batrla R, Klein C, Kramer M: Plasmin abrogates alpha $v$ beta 5-mediated adhesion of a human keratinocyte cell line (HaCaT) to vitronectin. Exp Cell Res 1995, 220:274-282.

23. Chun M: Plasmin induces the formation of multicellular spheroids of breast cancer cells. Cancer Lett 1997, I I 7:5 I-56.

24. Hannigan G, Troussard A, Dedhar S: Integrin-linked kinase: a cancer therapeutic target unique among its ILK. Nat Rev Cancer 2005, 5:5I-63.

25. Nista A, Leonetti C, Bernardini G, Mattioni M, Santoni A: Functional role of alpha 4 betal and alpha 5 beta $I$ integrin fibronectin receptors expressed on adriamycin-resistant MCF-7 human mammary carcinoma cells. Int J Cancer 1997, 72:133-14I.

26. Beketi(-Oreskovi( L, Sarcevi( B, Malenica B, Novak D: Immunocytochemical reactivity of a mouse monoclonal antibody CDI 3 I5B raised against human breast carcinoma. Neoplasma 1993, 40:69-74 